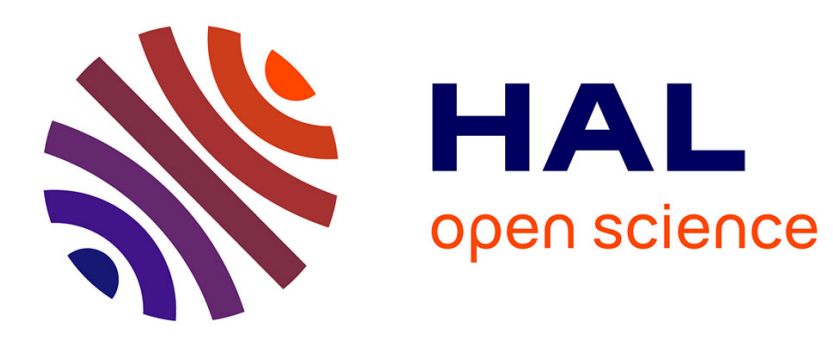

\title{
How do functionalized carbon nanotubes land on, bind to and pierce through model and plasma membranes.
}

Lara Lacerda, Hanene Ali-Boucetta, Sebastian Kraszewski, Mounir Tarek, Maurizio Prato, Christophe Ramseyer, Kostas Kostarelos, Alberto Bianco

\section{- To cite this version:}

Lara Lacerda, Hanene Ali-Boucetta, Sebastian Kraszewski, Mounir Tarek, Maurizio Prato, et al.. How do functionalized carbon nanotubes land on, bind to and pierce through model and plasma membranes.. Nanoscale, 2013, 5 (21), pp.10242-10250. 10.1039/c3nr03184e . hal-02558864

\author{
HAL Id: hal-02558864 \\ https://hal.science/hal-02558864
}

Submitted on 29 Apr 2020

HAL is a multi-disciplinary open access archive for the deposit and dissemination of scientific research documents, whether they are published or not. The documents may come from teaching and research institutions in France or abroad, or from public or private research centers.
L'archive ouverte pluridisciplinaire HAL, est destinée au dépôt et à la diffusion de documents scientifiques de niveau recherche, publiés ou non, émanant des établissements d'enseignement et de recherche français ou étrangers, des laboratoires publics ou privés. 


\section{Accepted Manuscript}

This article can be cited before page numbers have been issued, to do this please use: L. Lacerda, H. Ali-Boucetta, S. Kraszewski, M. Tarek, M. Prato, C. Ramseyer, K. Kostarelos and A. Bianco, Nanoscale, 2013, DOI: 10.1039/C3NR03184E.

\section{Nanoscale}

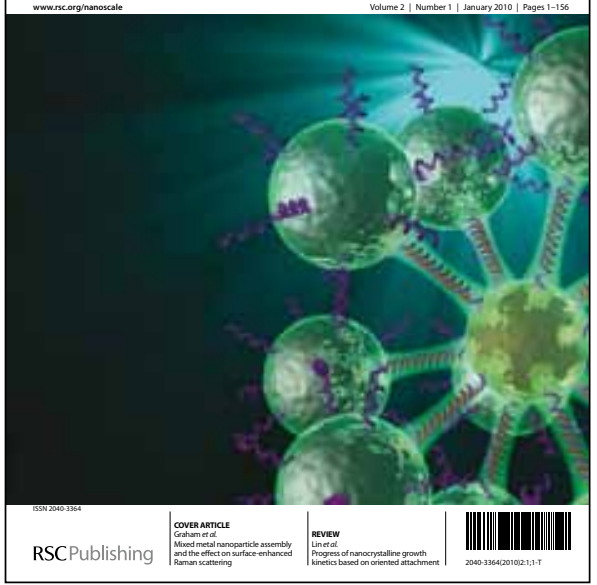

This is an Accepted Manuscript, which has been through the RSC Publishing peer review process and has been accepted for publication.

Accepted Manuscripts are published online shortly after acceptance, which is prior to technical editing, formatting and proof reading. This free service from RSC Publishing allows authors to make their results available to the community, in citable form, before publication of the edited article. This Accepted Manuscript will be replaced by the edited and formatted Advance Article as soon as this is available.

To cite this manuscript please use its permanent Digital Object Identifier $\left(\mathrm{DOl}^{\oplus}\right)^{\text {, }}$ which is identical for all formats of publication.

More information about Accepted Manuscripts can be found in the

\section{Information for Authors.}

Please note that technical editing may introduce minor changes to the text and/or graphics contained in the manuscript submitted by the author(s) which may alter content, and that the standard Terms \& Conditions and the ethical guidelines that apply to the journal are still applicable. In no event shall the RSC be held responsible for any errors or omissions in these Accepted Manuscript manuscripts or any consequences arising from the use of any information contained in them. 


\section{Table of Content Graphics}

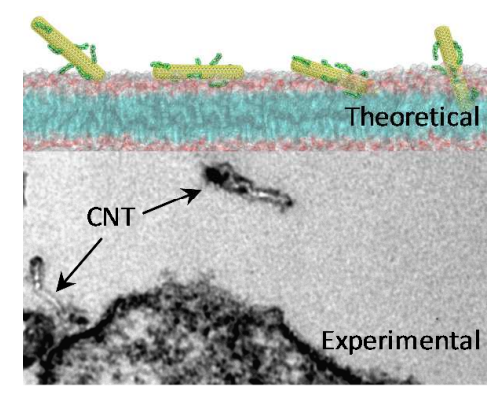

Experimental evidences and theoretical studies support that the cell interaction with plasma membrane and subsequent cell penetration of functionalized carbon nanotubes can occur in three distinct processes that can be summarized as landing, piercing, and uptake. 


\title{
How do functionalized carbon nanotubes land on, bind to and pierce through model and plasma membranes
}

\author{
Lara Lacerda, ${ }^{\mathrm{a}, \dagger}$ Hanene Ali-Boucetta, ${ }^{\mathrm{a}, \$}$ Sebastian Kraszewski, ${ }^{\mathrm{b}} *$ Mounir Tarek, ${ }^{\mathrm{c}}$ Maurizio Prato, ${ }^{\mathrm{d} *}$ \\ Christophe Ramseyer, ${ }^{b}$ Kostas Kostarelos, ${ }^{\mathrm{a}, *}$ and Alberto Bianco ${ }^{\mathrm{e}, *}$
}

Study of the mechanisms understanding how chemically functionalized carbon nanotubes internalize into mammalian cells is important in view of their design as new tools for therapeutic and diagnostic applications. The initial contact between the nanotube and the cell membrane allows elucidation of the types of interaction that are occurring and the contribution from the types of functional groups at the nanotube surface. Here we offer a combination of experimental and theoretical evidence of the initial phases of interaction between functionalized carbon nanotubes with model and cellular membranes. Both experimental and theoretical data reveal the critical parameters to determine direct translocation of the nanotubes through the membrane into the cytoplasm as a result of three distinct processes that can be summarized as landing, piercing and uptake.

\section{Introduction}

The study of mechanisms governing the cellular uptake of carbon nanotubes (CNTs) has been intensely addressed in the last few years. ${ }^{1-5}$ CNTs functionalized with different therapeutic molecules can readily cross cell membranes and distribute into the different cellular compartments following alternative routes. ${ }^{1,3}$ Two main mechanisms of uptake seem to be the prevailing ones: chemically functionalized CNTs ( $f$-CNTs) able to cross the cell membrane in a direct way, translocating into the cytoplasm, ${ }^{4}$ or an energetically active endocytotic/phagocytotic route. $^{3}$ These mechanisms are commonly coexisting during cell uptake and are strongly dependent on the type of nanotubes, the chemistry of functionalization, the charge and hydrophobic nature of the covalently linked molecules and the cell type. ${ }^{1}$ Importantly, utilization of the ability of CNTs to translocate directly into cells has opened the way to several studies exploring the potential of these nanomaterials to develop transport vectors for therapeutics and diagnostics. ${ }^{6,7}$

The first step during the process of cellular uptake involves the contact between the CNTs and the lipid bilayer constituting the cellular membrane. Different theoretical studies have attempted 
to elucidate the behavior of CNTs coming in contact with a cellular barrier using various models of simulation. ${ }^{8-13}$ These studies have been performed assuming pristine, non-functionalized CNTs or with nanotubes modified with small functional groups or biomolecules. Zimmerli et al. focused on the use of CNTs for the transportation of molecules inside the cells following the formation of transmembrane pores spanning the lipid bilayer. ${ }^{8}$ Liu et al. showed that a nanotube interacting with a cellular membrane can lead to lipid bilayer structural reorganization and alteration as a source of possible cytotoxity. ${ }^{9}$ Later, Yang et al. demonstrated that the cytotoxic effects caused by a membrane disruption can be mitigated by doping CNTs with nitrogen atoms. ${ }^{10}$ Recently, Kraszewski et al. described that nanotubes could land and float on the lipid bilayer, penetrating the lipid head-groups and sliding into the core of the membrane, ${ }^{11}$ such behavior being dependent on the length of the tube. ${ }^{12}$ This type of behavior is mostly driven by hydrophobic interactions as also shown for protein CNT unspecific binding. ${ }^{13-17}$ Short functionalized CNTs were also shown to have the propensity to pierce the lipid bilayer by a passive mechanism. ${ }^{12}$ In contrast, Skandani et al. reported that long nanotubes were able to translocate cell membranes faster, while short tubes underwent a rotation during the final step of uptake following an endocytotic route. ${ }^{18}$ It is evident that the characteristics of the nanotubes used in each theoretical simulation study can explain the differences found and reported, making difficult to determine the common principles that can govern CNTs cellular uptake based only on theoretical data. An attempt to correlate the theoretical studies with experimental evidence was recently reported by Shi et al. ${ }^{19}$ Pristine nanotubes seem to enter the cells via recognition of their tips by the membrane receptors, depending on the presence or the absence of the caps at the extremities of the tubes.

Another issue regarding CNTs and their interaction with model and cellular membranes is that the theoretical studies assume that it is impossible for CNTs to exit cells. ${ }^{12}$ However, experimental studies have demonstrated that CNTs can escape from the cytoplasm by exocytosis, mediated by cell-released microvesicles. ${ }^{20,21}$ Moreover, in cell-mock experiments conjugates formed by CNTs and small proteins, such as lysozymes, were able to induce liposome content leakage following the electrostatic interaction between the conjugate and the lipid membrane that was dependent on the liposome composition and the charge of the protein. ${ }^{22}$ Numerous factors control the interaction between CNT and model and cellular membranes and so far it is unclear which of these prevail at the initial step of interaction allowing successful binding and diffusion of CNT. The hallmark of theoretical simulation studies carried out to date is the corse-grain molecular dynamics work by Lopez et al. reporting the ability of amphiphilic organic (non- 
functionalized) nanotubes to penetrate a lipid bilayer in a so-called nanoneedle fashion. ${ }^{23}$ The aim of our study here was to offer new experimental elucidation of the initial steps of interaction between biocompatible $f$-CNTs (relevant to biomedical applications) with model and cellular membranes.

In order to understand the role of surface charge interactions between the functional groups attached to CNT and those on lipid bilayers, we determined the interaction between $f$-CNTs and liposomes (model membrane) using different experimental setups and monitoring the fluorescence spectrum and intensity of the membrane probe Nile Red. In addition, we examined $f$-CNTs in contact with a cell membrane at $4^{\circ} \mathrm{C}$ and $37^{\circ} \mathrm{C}$ by transmission electron microscopy (TEM) and finally performed an all-atom MD simulation study to describe such interactions at the atomic level.

\section{Results}

\subsection{Positively charged CNTs interact more with negatively DOPG liposomes}

In order to elucidate the binding and uptake mechanism of CNTs with mammalian cells, the interactions between water dispersible $f$-CNTs (single- and multi-walled) and model membranes (lipid bilayers) were first studied by fluorescence spectrophotometry. For this purpose, $f$-CNTs were prepared following the 1,3-dipolar cycloaddition reaction, which introduces positively charged amino groups onto the external wall of CNTs $\left(\mathrm{SWCNT}-\mathrm{NH}_{3}{ }^{+}\right.$and $\left.\mathrm{MWCNT}-\mathrm{NH}_{3}{ }^{+}\right)(\mathrm{Fig}$ 1). ${ }^{24,25}$ Part of the ammonium groups of nanotubes were modified by acetylation (SWCNT-NHAc) or by introducing diaminotriethylene pentacetic anhydride (SWCNT-DTPA), thus obtaining neutral and negatively charged CNTs, respectively (Fig. 1) (see ESI for the physico-chemical characteristics and TEM images of the different functionalized nanotubes).

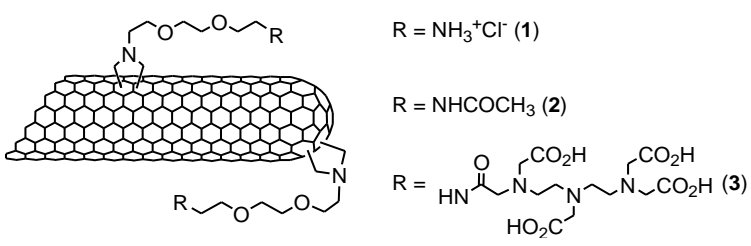

Figure 1.Molecular structures of the different $f$-CNTs. (1) $\mathrm{SWCNT}^{-\mathrm{NH}_{3}}{ }^{+}$or MWCNT-NH${ }_{3}{ }^{+},(2) \mathrm{SWCNT}-\mathrm{NH}-\mathrm{Ac}$, and (3) SWCNT-DTPA.

Lipid vesicles that consist of a mixture of phospholipids and cholesterol (Fig. 2) have been used as in vitro model membranes and mimic the interactions between peptides and drugs with cellular membranes. ${ }^{26,27}$ Such vesicles are commonly known as liposomes. In addition, the incorporation 
of small fluorescent molecules in liposomes has been used to study the bilayer microenvironment by techniques such as spectrophotometry. ${ }^{28,29}$

A

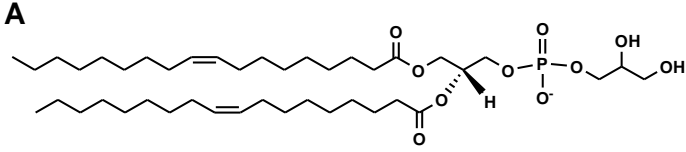

B

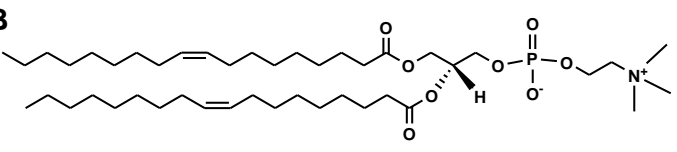

C

D

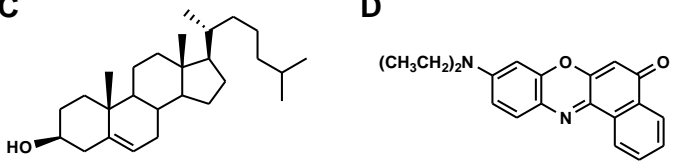

Figure 2.Molecular structures of the lipids and organic dye used. (A) Negatively charged phospholipid DOPG, (B) zwitterionic phospholipid DOPC, (C) cholesterol, and (D) membrane dye Nile Red.

In this study we used Nile Red (Fig. 2D), a photochemically stable, uncharged benzophenoxazone dye. Nile Red has a high partition coefficient from water to hydrophobic solvents. In addition, the presence of conjugated double bonds (delocalized electrons) allows its excitation with subsequent fluorescence emission. Due to its extremely hydrophobic characteristics, Nile Red fluoresces intensely when dissolved in organic solvents and lipids but its fluorescence is fully quenched in water. ${ }^{30-33}$ In order to confirm the sensitivity of Nile Red to changes in solvent's polarity and the resultant effecton its fluorescence characteristics, mixtures of chloroform/methanol (apolar/polar) were prepared at different volume ratios and Nile Red was added to each solvent mixture $\left(0.1 \mathrm{mg} \cdot \mathrm{ml}^{-1}\right)$. After scanning the fluorescence spectra of Nile Red diluted in different solvent dispersions, we observed that as the polarity of the solvent increases, the maximum fluorescence intensity of Nile Red shifts to higher wavelengths, from 600 to 637 $\mathrm{nm}$ and that the fluorescence intensity of Nile Red decreases by $60 \%$ of its maximum when dissolved in pure chloroform (Fig. 3). 


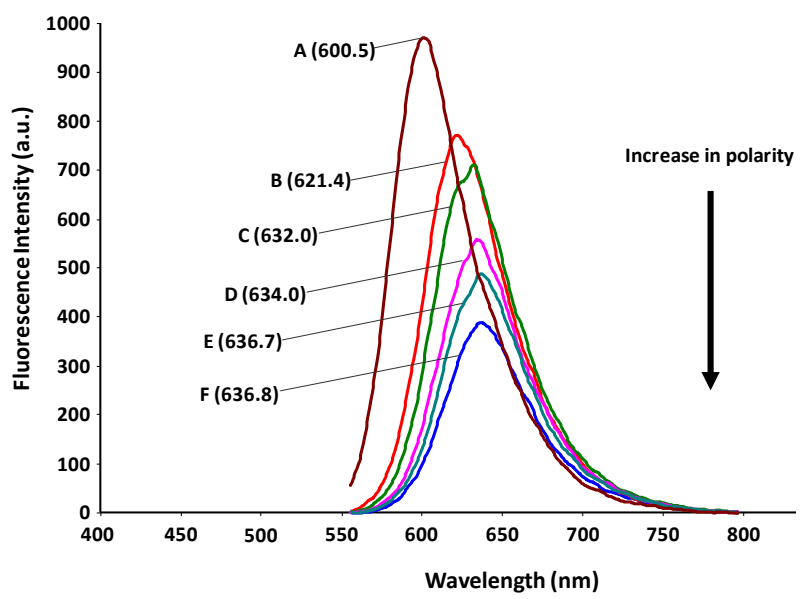

Figure 3. Effect of solvent polarity on fluorescence spectra of Nile Red. Fluorescence spectra of Nile Red dissolved in apolar/polar ( $\mathrm{vol} / \mathrm{vol}$ ) solvent mixtures of (A) chloroform only; (B) 8/2 chloroform/methanol; (C) 6/4 chloroform/methanol; (D) 4/6 chloroform/methanol; (E) 2/8 chloroform/methanol; (F) methanol only. The wavelength $(\mathrm{nm})$ for maximum intensity is indicated for each spectrum. Excitation wavelength was $542 \mathrm{~nm}$.

Following this preliminary experiment, we prepared large multilamellar vesicles (liposomes) by the lipid film hydration method. Two liposome formulations were prepared by changing the phospholipid used. DOPC was used to prepare neutral model membranes while DOPG generated negatively charged model membranes. Nile Red was added to each formulation in a ratio of 1:300, dye:lipid molecules. We assumed that the fluorescent probe was incorporated deeply into the lipid bilayer of the liposome, thus, its spectral characteristics would reflect the polarity of the model membrane microenvironment. As expected, and in agreement with the literature ${ }^{30,32}$ we observed that the fluorescence intensity of the dye was red-shifted (628 nm) (Fig. 4A), which indicates that the dye is not only incorporated into the membrane but is already in a more polar microenvironment than when dissolved in pure chloroform.

Subsequently, the interaction between $f$-CNTs and the model membranes was investigated using two differently charged multilamellar vesicles and several types of $f$-CNTs. Following addition of $f$-CNTs to aqueous suspensions of liposomes, each sample was kept for $30 \mathrm{~min}$ at room temperature to allow the interaction. Then, the fluorescence intensity of Nile Red was recorded (excitation wavelength of $542 \mathrm{~nm}$ ). The effect of a gradual addition of $f$-CNTs to Nile Redcontaining liposomes is shown in Figure 4. We observed that the fluorescence intensity of Nile Red incorporated into neutral DOPC membranes was reduced only $20 \%$ of the initial fluorescence intensity by the addition of SWCNT- $\mathrm{NH}_{3}{ }^{+}$(Fig. 4A). In contrast, the fluorescence intensity of Nile Red incorporated into negative DOPG membranes was reduced up to $75 \%$ of its initial value following the addition of $\mathrm{SWCNT}^{-\mathrm{NH}_{3}}{ }^{+}$(Fig. 4B). These results indicate that SWCNT- $\mathrm{NH}_{3}{ }^{+}$were able to change the lipid bilayer microenvironment in both types of vesicles. 
More importantly, positively charged SWCNT are able to interact with negatively charged lipid bilayers to a greater extent than with model membranes of neutral surface charge.

In parallel, experiments were carried out to investigate the effects of neutral (SWCNT-NH-Ac) and negatively (SWCNT-DTPA) charged nanotubes on the fluorescence intensity of Nile Red incorporated into neutral and negatively charged liposomes used as model membranes. The fluorescence intensity data were obtained, and the ratio $\mathrm{I} / \mathrm{I}_{0}$ (I, fluorescence reading after addition of $f$-CNTs; $\mathrm{I}_{0}$, fluorescence reading in the absence of $f$-CNTs) was calculated and plotted versus the amount of $f$-CNTs added to the aqueous suspension of lipid vesicles (Fig. 4C and D). As shown in Figure 4C, both SWCNT-NH-Ac and SWCNT-DTPA have moderate to no effect on Nile Red fluorescence intensity (modestly reduced (40\%) by SWCNT-NH-Ac and minimally decreased by SWCNT-DTPA), suggesting that the nanotubes interact to a limited extent with neutral membranes. Taken together, our results demonstrate that the interaction of $f$-CNTs with liposomes of opposite surface charge resulted in the most prominent decrease in Nile Red fluorescence intensity, indicating that positively charged nanotubes are able to remarkably disrupt the model membrane microenvironment of negatively charged model membranes.

In order to investigate the behaviour of larger (in diameter) $f$-CNTs $\left(\mathrm{MWCNT}_{\mathrm{NH}}{ }_{3}{ }^{+}\right.$) on both types of lipid vesicle model membranes, the fluorescence intensity changes of Nile Red incorporated into neutral and negatively charged lipid vesicles after the addition of MWCNT$\mathrm{NH}_{3}{ }^{+}$were analyzed. The interaction between nanotubes and vesicles of opposite surface charge resulted in the highest reduction in fluorescence intensity (about 70\%, Fig. 4C and D). 

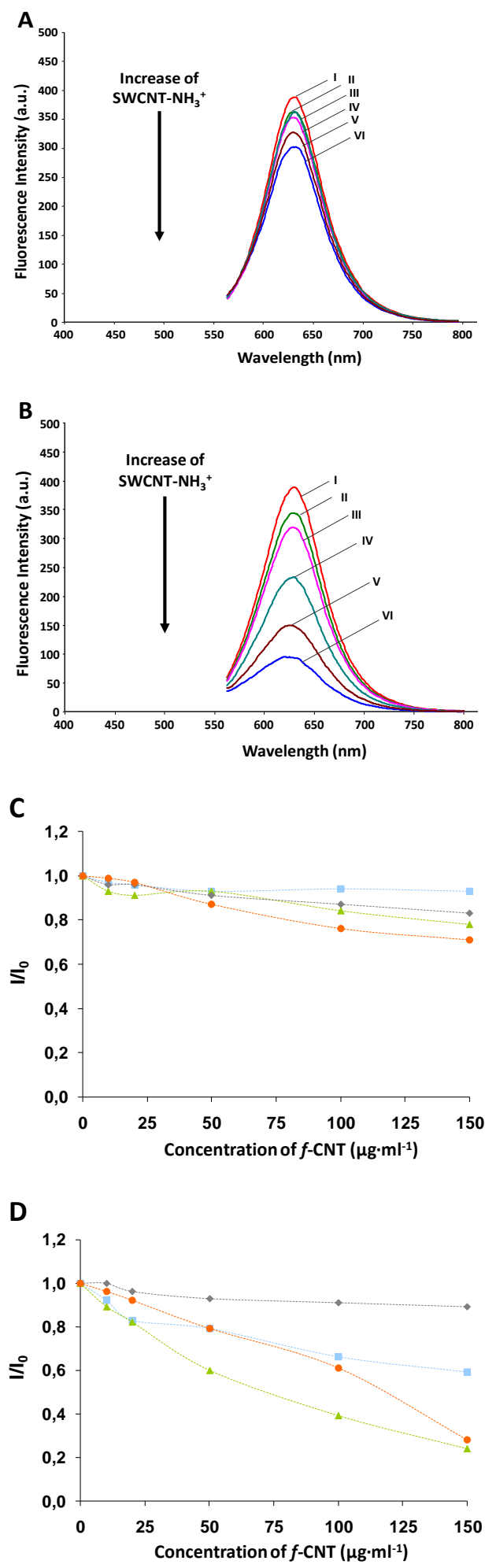

Figure 4. Interaction of $f$-CNTs with lipid model membranes. Fluorescence spectra of Nile Red embedded in lipid bilayers exposed to increasing quantities of SWCNT-NH ${ }_{3}{ }^{+}$(spectrum I to VI: $0,10,20,50,100$ and $150 \mu \mathrm{g} \cdot \mathrm{ml}^{-1}$ ): (A) DOPC liposomes; (B) DOPG liposomes. Fluorescence intensity ratio of Nile Red embedded in lipid bilayers exposed to increasing quantities (0 to $150 \mu \mathrm{g} / \mathrm{ml}$ ) of $f$-CNTs: (C) DOPC liposomes, and (D) DOPG liposomes. Solutions were excited at $542 \mathrm{~nm}$ and fluorescence peak intensity values were registered at $628 \mathrm{~nm}$. Grey diamond:

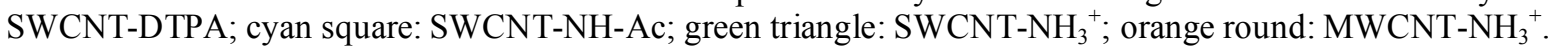


These results demonstrate that electrostatic interactions play a critical role in the initial contact between $f$-CNTs and model membranes. Positively charged $\mathrm{f}$-CNTs interacted consistently much stronger with negatively charged DOPG lipid vesicles compared to other types of $f$-CNTs leading to much more significant lipid bilayer perturbations. Overall, it needs to be noted that all $f$-CNTs used in this study exhibited a degree of interaction with neutral DOPC vesicles.

\section{2 $f$-CNT spatial orientation and binding to cell membranes independent of temperature}

In order to understand this initial interaction between $f$-CNTs and the plasma membrane further, a series of experiments using TEM imaging were performed, to study the orientation and spatial conformation of the nanotubes as they approach and come in contact with cellular membranes. MWCNT- $\mathrm{NH}_{3}{ }^{+}$were incubated with human lung epithelial (A549) cells for periods from 15 to $120 \mathrm{~min}$ at the physiologically relevant temperature of $37^{\circ} \mathrm{C}$. No cytotoxic effects such as cell detachment or blebbing were observed with the concentration of MWCNT-NH${ }_{3}^{+}$used in this study. After fixing and embedding the cells into EPON resin, ultra-thin sections of the cell cultures were prepared and examined by TEM. Figure 5 shows representative images of MWCNT $-\mathrm{NH}_{3}{ }^{+}$in the process of interacting with the plasma membrane.

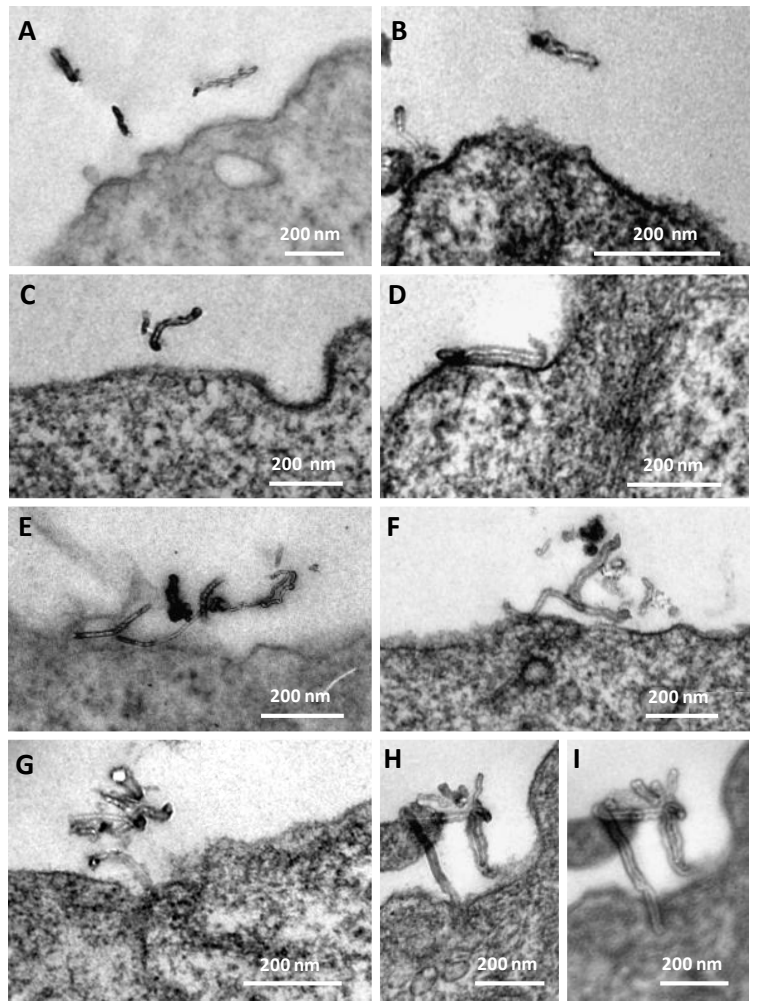

Figure 5. Interaction of MWCNT- $\mathrm{NH}_{3}{ }^{+}$with plasma membranes at $37{ }^{\circ} \mathrm{C}$. TEM images of $\mathrm{MWCNT}-\mathrm{NH}_{3}{ }^{+}$ incubated with A549 cells at $37{ }^{\circ} \mathrm{C}$. (A-C) $\mathrm{MWCNT}-\mathrm{NH}_{3}{ }^{+}$approaching the membrane, (D-F) $\mathrm{MWCNT}^{-\mathrm{NH}_{3}}{ }^{+}$ binding to the membrane, and (G-I) MWCNT- $\mathrm{NH}_{3}{ }^{+}$crossing the membrane. Image I is an out-off focus image of $\mathrm{H}$, to show the MWCNT- $\mathrm{NH}_{3}{ }^{+}$structure beyond the plasma membrane. 
Nanotubes reach the surface of the cell membrane in two types of orientations: either in parallel or perpendicular to the axis of the membrane (Fig. 5A-C). However, MWCNT- $\mathrm{NH}_{3}{ }^{+}$seem to bind preferentially to the membrane with their longitudinal axis in parallel to the contact surface (Fig. 5D-F). These results suggest that the orientation of the CNT axis in parallel to that of the membrane offers higher surface area, with more positive functional groups able to establish stronger electrostatic interactions, and overall better bind the nanotubes to the negatively charged cellular membrane.

In addition, the interaction of MWCNT- $\mathrm{NH}_{3}{ }^{+}$with the plasma membrane was also observed at a later stage, when the nanotubes had already inserted their tip through the membrane (Fig. 5G-I). These images demonstrate that these chemically functionalised nanotubes have the ability to reorient vertically to the membrane axis, following their initial contact in a parallel orientation. In order for $f$-CNTs to be able to translocate the plasma membrane, they appear to adopt a perpendicular orientation in relation to the plasma membrane. As confirmed by the out-of-focus images (Fig. 5I), the intact nanotubes were able to pierce the cell membrane, as their tips could be seen to insert through the bilayer and into the cytoplasm. Such observations are in agreement with previous work that reported $f$-CNTs capable of translocating intact through the plasma membranes and into the cytoplasm. ${ }^{4,34,35}$

In order to determine whether the spatial orientation between $f$-CNT and cell membranes is energy-dependent, MWCNT- $\mathrm{NH}_{3}{ }^{+}$were incubated with $\mathrm{A} 459$ cells also at $4{ }^{\circ} \mathrm{C}$ and studied by TEM (Fig. 6A-C).

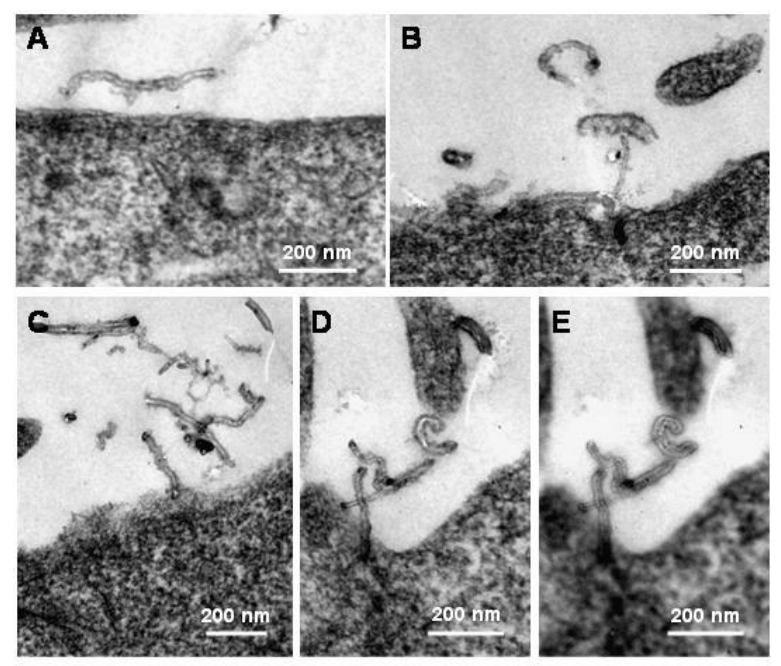

Figure 6. Interaction of MWCNT- $\mathrm{NH}_{3}{ }^{+}$with plasma membranes at $4{ }^{\circ} \mathrm{C}$. TEM images of MWCNT-NH${ }^{+}$incubated with A549 cells at $4{ }^{\circ} \mathrm{C}$. (A-C) MWCNT- $\mathrm{NH}_{3}{ }^{+}$approaching and binding the membrane, and (D \& E) MWCNT-NH ${ }^{+}$ crossing the membrane. Image $\mathrm{E}$ is an out-off focus image of $\mathrm{D}$, to show the $\mathrm{MWCNT}-\mathrm{NH}_{3}{ }^{+}$structure beyond the plasma membrane. 
Nanotubes were found to bind to membranes and also seen to reorient to a spatial conformation perpendicular to that of the plasma membrane, able to also insert their tip through the membrane and into the cytoplasm (Fig. 6D and E). These results further indicate that $\mathrm{MWCNT}-\mathrm{NH}_{3}{ }^{+}$ interaction and orientation in relation to plasma membranes are independent of temperature.

\section{3 $f$-CNT passive diffusion in three steps: landing, piercing and translocation}

The experimental studies described above were complemented by simulations of functionalized SWCNTs $(\sim 1 \mathrm{~nm}$ in diameter and $\sim 5 \mathrm{~nm}$ in length) with a model membrane. We have investigated using all-atom molecular dynamics (MD) simulations the interactions between amino-functionalized SWCNTs and a fully hydrated POPC membrane. This theoretical method is one of the most efficient ways to reveal the molecular mechanisms at initiating CNT cellular uptake. The model nanotube contains seven amino groups (one every 86 carbon atoms) randomly distributed onto the CNT surface, thus directly agreeing with the experimental $f$-CNT (1) shown in Figure 1 (similar degree of functionalization). Representative snapshots of MD runs illustrated $f$-CNT translocation by passive diffusion as can be seen in Figure 7.

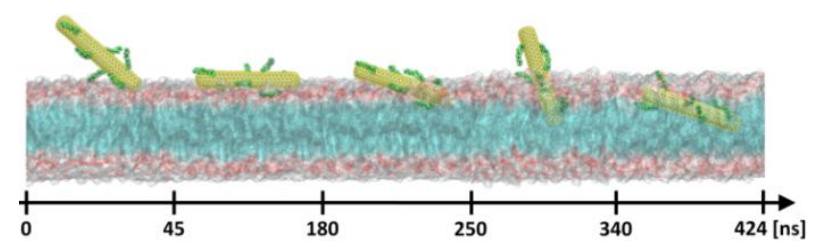

Figure 7. Consecutive snapshots issued from unconstrained MD simulation of $f$-CNT diffusing passively to the lipid membrane. Molecular mechanism of interactions can be divided on three main processes: 1) landing (up to $180 \mathrm{~ns}$ ), 2) nanoneedle like picking (up to $340 \mathrm{~ns}$ ), and 3) uptake.

Such diffusion can be separated in three distinct processes: 1) surface landing, 2) membrane piercing, and 3) translocation. First, $f$-SWCNT approached the membrane at an angle $\alpha$ of about $20^{\circ}$ with respect to the normal direction of the bilayer plane (Fig. 8A). After $30 \mathrm{~ns}$, landing occurred and $f$-CNT remained parallel to the membrane interface for over $180\left(\alpha=90^{\circ}\right)$. At $\sim 200$ ns, the $f$-SWCNT reoriented with respect to the membrane at an angle $\sim 15^{\circ}$ then, as a nanoneedle, dove into its hydrophobic core dragging there water molecules (Fig. 8B). Finally, the $f$-SWCNT was uptaken into the membrane core (at $\sim 340 \mathrm{~ns}$ ) and stayed in this configuration until the end of simulation (424 ns). The amount of water molecules present in hydrophobic core of membrane increased locally of about $40 \%$ during $f$-SWCNT insertion. This can be at the origin of membrane 
disruption. Detailed illustration of the $f$-SWCNT insertion process into lipid bilayer can be also seen in the video provided in the Supplementary Material.

From the simulation performed, the complete translocation of intact $f$-SWCNT throughout the lipid bilayer was not able to be observed. This was probably due to the charge interactions between the functional parts of the CNT and the lipid heads. It can be assumed that for $f$-SWCNT to translocate intact through the whole plasma membrane a deprotonation/reprotonation mechanism should occur similar to that previously described for charged amino-functionalized fullerenes. ${ }^{31}$ Our MD did not incorporate such deprotonation/reprotonation mechanisms.
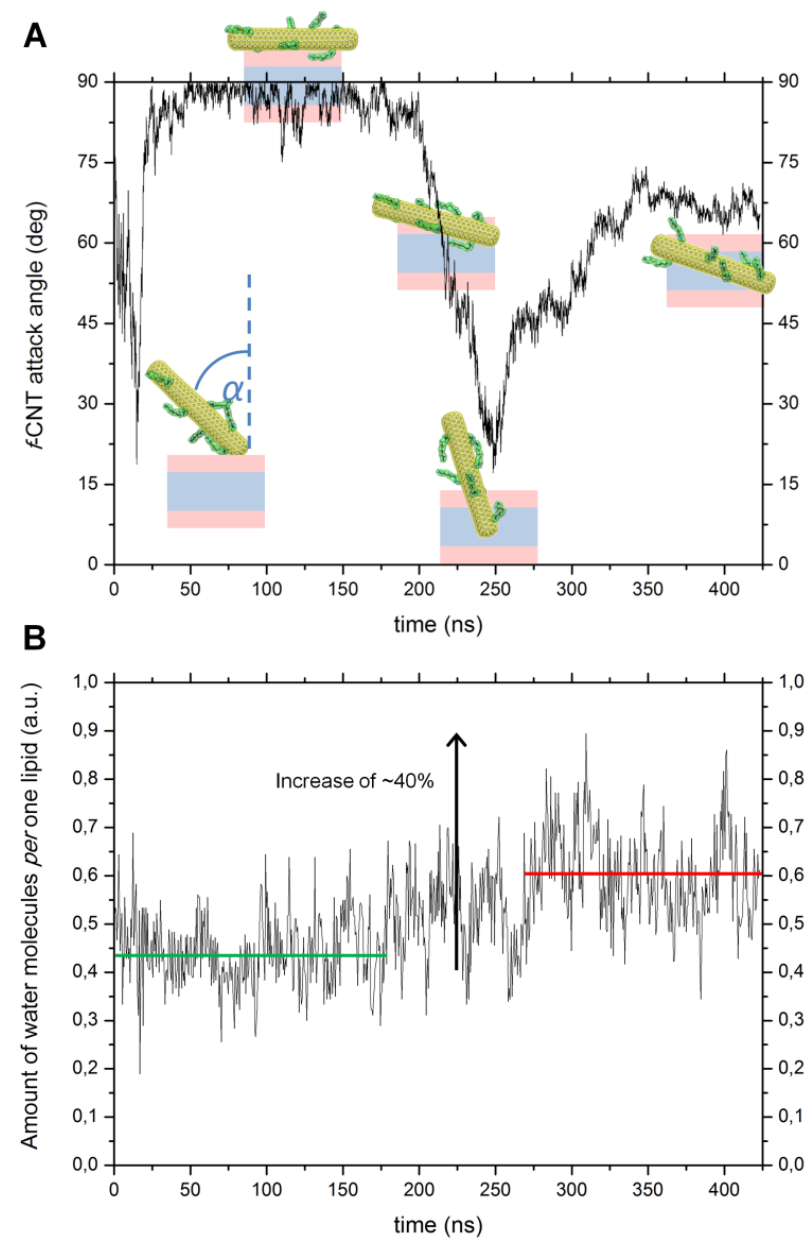

Figure 8. Behavior of water and $f$-CNT during lipid insertion. (A) $f$-SWCNT attack angle $(\alpha)$ with respect to the normal of the membrane plane, as a function of simulation time during its translocation. (B) Amount of water molecules present in hydrophobic core of membrane increases from 0.43 (per one lipid molecule) to about 0.60 during $f$-SWCNT insertion.

\section{Discussion}

Although several studies have reported the internalization of CNTs by eukaryotic and mammalian cells, the initial interactions, the factors determining such interactions and how the 
CNTs enter cells are still unclear and mainly addressed by theoretical calculations. ${ }^{8-19}$ In this study we have attempted to correlate the experimentally determined binding of $f$-CNTs with model membranes (lipid vesicles) and plasma membranes (A549 cells) with the interactions obtained by molecular dynamics simulations. We conclude that electrostatic interactions between the hydrophilic and charged functional moieties of $f$-CNTs and the polar headgroups of the lipid vesicles are the major contributors to the overall initial interaction.Furthermore, our experimental observations were found to be in good agreement with theoretical work by Lopez et al. showing that an amphiphilic nanotube (3 $\mathrm{nm}$ length and $1.3 \mathrm{~nm}$ diameter) with hydrophilic functional groups localized at its terminal parts spontaneously insert into a lipid bilayer in a two-step mechanism: (a) the nanotube is adsorbed onto the membrane surface, and (b) it reorients spontaneously and adopts a transmembrane configuration. ${ }^{23}$ Our data are also in accord with those recently reported by Shi et al. ${ }^{19}$ These authors attempted to correlate the risks of CNT length-dependent toxicity to the mechanisms of cell uptake to design safer nanotubes. ${ }^{37}$ The interactions of non-functionalized multi-walled carbon nanotubes with cell membranes were elucidated experimentally and theoretically. Similarly to our results, the nanotubes enter the cells through the tip. In particular, the tips of nanotubes with closed extremities are initially recognized by and bind to cell membrane receptors, subsequently leading to a rotation of the tube and an almost vertical entry. ${ }^{19}$

The reduction in the fluorescence intensity of Nile Red incorporated into our DOPG model membrane indicate that the bilayer environment was more polar after the gradual addition of positively charged CNTs to the negatively charged membrane. Thus, the increase of polarity within the bilayer microenvironment is likely due to the interaction, binding and eventual piercing of the $f$-CNTs through the model membrane. For these reasons, we concluded that CNT$\mathrm{NH}_{3}{ }^{+}$interact primarily through attractive electrostatic forces to approach and adsorb onto model membranes. However, if the hydrophobic dye is deeply incorporated in the bilayer, further events must develop after the binding step to promote the quenching of the dye. We speculate that the $f$ CNTs penetrated the lipid membrane and caused a concomitant introduction of water molecules in the lipid bilayer hydrophobic space that increased its polarity.

Earlier we have shown that the cellular uptake of functionalized carbon nanotubes is independent of functional group and cell type. ${ }^{4}$ In the present work, studies on model membranes were complemented by TEM studies of the initial interactions of nanotubes with plasma membranes using mammalian cells. We observed that $\mathrm{MWCNT}-\mathrm{NH}_{3}{ }^{+}$initially interacted with cell membranes with their long axis in parallel to the plasma membrane axis, but later they reoriented 
perpendicular to the plasma membrane and inserted their tips through. Furthermore, our results showed that the process of $f$-CNTs diffusion through cell membranes was not dependent of the temperature that cells were incubated with $f$-CNT. Such results are in agreement with previously published experimental $^{1,4,38}$ and theoretical ${ }^{11}$ work that showed $f$-CNTs able to pierce cell membranes by passive diffusion.

Lastly, the MD simulation performed indicated that CNT diffusion through membranes can occur in three distinct processes: 1) landing, 2) piercing, and 3) uptake, that summarized all the experimental work described.In conclusion, the present studies provided experimental evidence confirmed by MD simulations, suggesting that chemically functionalized, biocompatible CNTs must have hydrophilic and positively-charged moieties in order to maximize interactions and bind with the moderate negatively-charged plasma membranes. Following binding, the nanotubes are shown able to reorient perpendicular to the axis of the membrane and finally allow hydrophobic interactions to pierce the lipid bilayers of the membranes that can lead to translocation of the nanotube. Overall, a fine balance between surface charge characteristics of the nanotube appended functional groups and the exposure of the hydrophobic domains of the carbon backbone seem to be responsible for the molecular interactions with plasma membrane components that can lead to their direct translocation through the membrane and into the cytoplasm.

\section{Experimental section}

\subsection{CNTs}

Pristine HiPco SWCNTs were purchased from Carbon Nanotechnologies Inc. (Lot No.: R0496). According to the manufacturer, the mean diameter of SWCNTs is about $1 \mathrm{~nm}$. Tubes have lengths between 300-1000 nm. Pristine MWCNTs were obtained from Nanostructured and Amorphous Materials Inc. (Lot No.: 1240XH, 94\% pure, outer diameters between 20 and 30 nm, and lengths between 0.5-2 $\mu \mathrm{m}$ ).

\subsection{Functionalization of CNTs}

Single- and multi-walledammonium-functionalized CNTs $\left(\mathrm{SWCNT}_{-} \mathrm{NH}_{3}{ }^{+}\right.$and $\mathrm{MWCNT}-\mathrm{NH}_{3}{ }^{+}$) were synthesized by 1,3-dipolar cycloaddition reaction as previously reported. ${ }^{24,25}$ Acetamidofunctionalized CNT (SWCNT-NH-Ac) were prepared as described in reference. ${ }^{39}$ DTPAfunctionalized CNT (SWCNT-DTPA) were prepared as reported in reference. ${ }^{40}$

Following the functionalization by the 1,3-dipolar cycloaddition reaction, CNTs with ammonium moieties were readily soluble in deionized water at relatively high concentrations $(5-10 \mathrm{mg} / \mathrm{ml})$. 
Additional modification with acetyl groups slightly reduced the solubility of CNTs, while the modification with DTPA did not change the solubility of CNTs compared the precursor nanotubes with ammonium groups. Stock dispersions were prepared by hydration of each type of $f$-CNTs in deionized water at concentrations of $6.7 \mathrm{mg} / \mathrm{ml}\left(\mathrm{MWCNT}-\mathrm{NH}_{3}{ }^{+}\right), 6 \mathrm{mg} / \mathrm{ml}(\mathrm{SWCNT}$ $\mathrm{NH}_{3}{ }^{+}$) and $3 \mathrm{mg} / \mathrm{ml}$ (SWCNT-Ac and SWCNT-DTPA) under mild sonication using a water bath. All dispersions were kept at $4^{\circ} \mathrm{C}$ until needed and always sonicated before used.

\subsection{Model membrane (liposome) preparation}

Liposomes were prepared by the lipid film hydration method. Briefly, phospholipids DOPG [1,2dioleoyl-sn-glycero-3-[phospho-rac-(1-glycerol), from Sigma] and DOPC (1,2-dioleoyl-snglycero-3-phosphocholine, from Avanti) and cholesterol (Sigma) were dissolved in chloroform/methanol (4:1 vol/vol) at a final total lipid concentration of $2 \mathrm{mM}$ of which $10 \mathrm{~mol} \%$ was cholesterol. Nile Red (membrane probe) was added to the lipid mixture in a ratio of 1:300 lipid molecules. After evaporation of the organic solvents, the lipid film was hydrated with $1 \mathrm{~mL}$ saline solution $(0.09 \% \mathrm{NaCl})$ and vortexed for $15 \mathrm{~min}$ to achieve a final concentration of $2 \mathrm{mM}$. Following hydration, large multilamillar liposomes were obtained. All liposome suspensions were kept at $4{ }^{\circ} \mathrm{C}$ until needed.

\subsection{Fluorescence intensity studies}

$f$-CNTs were added to $50 \mu \mathrm{g}$ of liposomes (DOPG and DOPC) and rapidly mixed. The suspension mixtures were incubated at room temperature for $30 \mathrm{~min}$ and then fluorescence emission of Nile Red was scanned on a Perkin Elmer LS 50B spectrophotometer. Quartz cuvettes with a path length of $4 \mathrm{~cm}$ were used. Samples were excited at $542 \mathrm{~nm}$ and emission scans were recorded from 550 to $800 \mathrm{~nm}$.

\subsection{Transmission electron microscopy studies}

In order to investigate the cellularinteraction of $f$-MWCNTs by transmission electron microscopy (TEM), A549 cells were seeded in Millicell ${ }^{\circledR}$ plate inserts $(12 \mathrm{~mm}$, polycarbonate membrane, 0.4 $\mu \mathrm{m}$ pore size), 3 days prior the experiment. Then, after being washed with PBS, the cells were incubated with MWCNT-NH${ }_{3}^{+}\left(50 \mu \mathrm{g} \cdot \mathrm{ml}^{-1}\right)$ at $37^{\circ} \mathrm{C}$ or $4^{\circ} \mathrm{C}$ for $15,30,60$ and 120 min in serumfree media. Cellswerethen rewashed with PBS and fixed with $3 \%$ glutaraldehyde in $0.1 \mathrm{M}$ cacodylate buffer ( $\mathrm{pH} 7.4$ ) at $4^{\circ} \mathrm{C}$ overnight. On the following day, after washing the cells 3 times with distilled water, a secondary fixation was carried out with $1 \%$ aqueous osmium tetroxide $\left(\mathrm{OsO}_{4}\right)$ for $60 \mathrm{~min}$. The cells were then rinsed with water and dehydrated in a series of ethanol $(70 \%, 90 \%$ and $100 \%)$. Infiltration with araldite resin (Agar, UK) was done using a 1:1 mixture of ethanol and araldite resin mix (2 times for 15 minutes each) and neat resin $(2 \mathrm{~h})$. The wells 
were filled with fresh resin and placed into the oven to polymerise the resin at $60^{\circ} \mathrm{C}$ for $48 \mathrm{~h}$. Ultra-thin sections (65-85 nm thick) were stained with uranyl acetate and lead citrate, and imaged with a Philips CM10 TEM at $80 \mathrm{kV}$. Images were captured with a high resolution digital camera.

\subsection{Molecular dynamics simulations}

Unconstrained full atomistic molecular dynamics (MD) simulations were performed using an equilibrated, fully hydrated zwitterionic POPC (palmitoyl-oleoyl-phosphatidylcholine) membrane model. At the temperature set for the study, i.e. $300 \mathrm{~K}$, the bilayer is in the biologically relevant liquid-crystalline $(\mathrm{L} \alpha)$ phase. The simulation box consists of 180 POPC units, 14512 water molecules and one water-soluble closed $(6,6)$-SWCNT $(\sim 1 \mathrm{~nm}$ in diameter and $\sim 5 \mathrm{~nm}$ in length). Such a small length of $f$-CNT was chosen to perform a quantitative simulation witin a sufficient timeframe (424 ns). The functionalized CNT contains 7 ammonium groups $\left(f-\mathrm{SWCNT}-\mathrm{NH}_{3}{ }^{+}\right)$randomly distributed on the whole surface (one functional group per 86 carbon atoms). The initial dimensions of the simulation box of $7.0 \times 7.4 \times 12.6 \mathrm{~nm}^{3}$ were chosen to ensure a reasonable thickness of the water layers on both sides of the membrane $(8.6 \mathrm{~nm}$ taking into account periodic boundary conditions). This is also large enough to accommodate a $5.3 \mathrm{~nm}$ long $f$-CNT which was used in the MD study. The system neutrality was guaranteed by adding 7 chlorine counter ions. All MD simulations have been carried out using NAMD2.6, ${ }^{41}$ a program targeted for massively parallel architectures. Short- and long-range forces were calculated every 1 and 2 time-steps respectively, with an integration time of 2.0 fs. The Langevin dynamics algorithm and the Langevin piston Nosé-Hoover method ${ }^{42}$ were used to maintain constant $300 \mathrm{~K}$ temperature and 1 bar pressure in the system. Long-range electrostatic forces were taken into account using the particle mesh Ewald (PME) approach. ${ }^{43}$ The force field parameters for lipid are taken from CHARMM $27^{44}$ with the united atoms extension. The intra- and intermolecular potentials for water are modeled within the TIP3P approach. ${ }^{45}$ For the CNT carbon-carbon or carbon-water interactions, we followed the Bedrov's description ${ }^{46}$ (CHARMM27 functionals: $\sigma \mathrm{CC}=3.895 \AA, \varepsilon \mathrm{CC}=0.066 \mathrm{kcal} \cdot \mathrm{mol}^{-1}$ and $\left.\sigma \mathrm{CO}=3.580 \AA, \varepsilon \mathrm{CO}=0.0936 \mathrm{kcal} \cdot \mathrm{mol}^{-1}\right)$. Necessary interaction parameters of the ammonium group (electrostatics and force constants) were extracted from ab initio calculations. We used the Gaussian03 package software ${ }^{47}$ to evaluate the effective electrostatic potential. The RHF//6-31+G level of theory was considered together with the polarized continuum water model (integral equation formalism IEFPCM) in order to reproduce the environmental effect of the solvent. Supplementary analysis based on construction of the Hessian matrix (the matrix of second derivatives of the energy with respect to geometry) was also performed to extract the force constants between atoms in the ammonium group. The derived 
partial charges (Mulliken charges distributions) ${ }^{48}$ were then applied in the MD simulations, accordingly to the results obtained from the quantum mechanics calculations. Following quantum mechanical calculations a large scale MD simulation was performed. First, the stability of $f$-CNT was tested in the water box in order to find a stable equilibrated structure before inserting it to the system containing hydrated POPC lipid bilayer. Next, $f$-CNT was placed in the bulk water parallel and not closer than $5 \AA$ to the membrane surface.

\section{Acknowledgments}

This work was supported by CNRS (PICS 2010 Program), University of Trieste and Italian Ministry of Education MIUR (cofin Prot. 2010N3T9M4 and Firb RBAP11C58Y). Partial funding from Biotechnology and Biological Sciences Research Council (BB/J008656/1) is also acknowledged. A. Battigelli is grateful to Università italo-francese/Université franco-italienne for supporting her international PhD (VINCI program 2008). L. L. acknowledges support by the Portuguese Foundation for Science and Technology (FCT/MCTES) for the award of a PhD fellowship (Ref. SFRH/BD/21845/2005).

\section{Notes and references}

${ }^{a}$ Nanomedicine Lab, School of Medicine \& National Graphene Institute, Faculty of Medical and Human Sciences, AV Hill Building, University of Manchester, Manchester M13 9PT, United Kingdom.E-mail: kostas.kostarelos@manchester.ac.uk

${ }^{b}$ Laboraoire de Nanomédecine, Imagerie et Thérapeutiques, EA 4662, Université de FrancheComté, Centre Hospitalier Universitaire de Besançon, 25000 Besançon, France. E-mail: sebastian.kraszewski@univ-fcomte.fr ${ }^{c}$ UMR Structure et Réactivité des Systèmes Moléculaires Complexes, Nancy-University, CNRS, France.

${ }^{d}$ Dipartimento di Scienze Chimiche e Farmaceutiche, Università di Trieste, Piazzale Europa 1, 34127 Trieste, Italy. E-mail: prato@units.it

${ }^{e}$ CNRS, Institut de BiologieMoléculaire et Cellulaire, Laboratoired'Immunopathologie et ChimieThérapeutique, UPR 3572, Strasbourg, 67000, France. E-mail: a.bianco@ibmccnrs.unistra.fr 
${ }^{[\dagger]}$ Current address: Department of Radiation Oncology, The University of MD Anderson Cancer Center, 1515 Holcombe Blvd., Houston, TX 77030, USA.

${ }^{[\$]}$ Current address: Pharmacy, Pharmacology and Therapeutics, School of Experimental and Clinical Medicine, Medical School, University of Birmingham, Birmingham B15 2TT, UK.

$\dagger$ Electronic Supplementary Information (ESI) available: CNT physico-chemical characteristics, TEM images and simulation video. See DOI:

\section{References}

1 L. Lacerda, J. Russier, G. Pastorin, M. A. Herrero, E. Venturelli, H. Dumortier, K.T. Al-Jamal, M. Prato, K. Kostarelos, and A. Bianco, Biomaterials, 2012, 33, 3334.

2 V. Raffa, G. Ciofani, O. Vittorio, C. Riggio and A. Cuschieri, Nanomedicine (Lond), 2010, 5, 89.

3 N.W. Kam, Z. Liu and H. Dai, Angew. Chem. Int. Ed., 2006, 45, 577.

4 K. Kostarelos,L. Lacerda, G. Pastorin, W. Wu, S. Wieckowski, J. Luangsivilay, S. Godefroy, D. Pantarotto, J.-P. Briand, S. Muller, M. Prato and A. Bianco, Nat. Nanotech., 2007, 2, 108.

5 M.H. Lamm and P.C. Ke, Meth. Mol. Biol., 2010, 625, 135.

6 K. Kostarelos, A. Bianco and M. Prato, Nat. Nanotech., 2009, 4, 627.

7 Z. Liu, S. Tabakman, K. Welsher and H. Dai, Nano Res., 2009, 2, 85.

8 U. Zimmerli and P. Koumoutsakos, Biophys. J., 2008, 94, 2546.

9 J. Liu and A. Hopfinger, J. Chem. Res. Toxicol., 2008, 21, 459.

10 Y. Yang, X. Li, J. Jiang, H. Du, L. Zhao and Y. Zhao, ACS Nano, 2010, 4, 5755.

11 S. Kraszewski, A. Bianco, M. Tarek and C. Ramseyer, PloS ONE 2012, e40703.

12 S. Kraszewski, F. Picaud, I. Elhechmi, T. Gharbi and C. Ramseyer, Carbon, 2012, 50, 5301.

13 J.-W. Shen, T. Wu, Q. Wang and Y Kang, Biomaterials, 2008, 29, 3847.

14 G. Zuo, Q. Huang, G. Wei, R. Zhou and H. Fang, ACS Nano, 2010, 4, 7508.

15 G. Zuo, W. Gu, H. Fang and R. Zhou, J. Phys. Chem. C, 2011, 115, 12322.

16 M. Calvaresi, S. Hoefinger and F. Zerbetto, Chem. Eur. J., 2012, 18, 4308.

17 F. De Leo, J. Sgrignani, D. Bonifazi and A. Magistrato, Chem. Eur. J., doi:10.1002/chem.201301376

18 A. AlipourSkandani, R. Zeineldin and A. Al-Haik, Langmuir, 2012, 28, 7872.

19 X. Shi, A. von den Bussche, R.H. Hurt, A.B. Kane and H. Gao, Nat. Nanotech., 2011, 6, 714.

20 H. Jin, D.A. Heller and M.S. Strano, Nano Lett., 2008, 8, 1577.

21 I. Marangon, N. Boggetto, C. Ménard-Moyon, E. Venturelli, M.-L. Beoutis, C. Péchoux, N. Luciani, C.

Wilhelm, A. Bianco and F. Gazeau,Nano Lett., 2012, 12, 4830.

22 A. Hirano, K. Uda, Y. Maeda, T. Akasaka and K. Shiraki, Langmuir, 2010, 26, 17256. 
23 C.F. Lopez, S.O. Nielsen, P.B. Moore and M.L. Klein, Proc. Natl. Acad. Sci. USA, 2004, 101, 4431.

24 V. Georgakilas, K. Kordatos, M. Prato, D. M. Guldi, M. Holzinger, and A. Hirsch, J. Am. Chem. Soc., 2002, 124, 760.

25 V. Georgakilas, N. Tagmatarchis, D. Pantarotto, A. Bianco, J.-P. Briand and M. Prato, Chem. Commun., 2002, 3050.

26 F. Reig, I. Haro, D. Polo, M.A. Egea and M.A. Alsina, J. Coll. Interf. Sci., 2002, 246, 60.

27 M. Kepczynski, A. Karewicz, A. Gornicki and M. Nowakowska, J. Phys. Chem. B, 2005, 109, 1289.

28 E. Perochon, A. Lopez, J.F. Tocanne, Biochemistry, 1992, 31, 7672.

29 A.S. Klymchenko, G. Duportail, A.P. Demchenko and Y. Mely, Biophys. J., 2004, 86, 2929.

30 P. Greenspan and S.D. Fowler, J. Lipid Res., 1985, 26, 781.

31 A.K. Dutta, K. Kamada and K. Ohta, J. Photoch. Photobio. A, 1996, 93, 57.

32 K. Kostarelos, P.F. Luckham and T.F. Tadros, J. Coll. Interf. Sci., 1997, 191, 341.

33 M.M.G. Krishna, J. Phys. Chem. A, 1999, 103, 3589.

34 D. Pantarotto, R. Singh, D. McCarthy, M. Erhardt, J.-P. Briand, M. Prato, K. Kostarelos and A. Bianco, Angew. Chem. Int. Ed. Engl., 2004, 43, 5242-5246.

35 J. Rojas-Chapana, J. Troszczynska, I. Firkowska, C. Morsczeck and M. Giersig, Lab Chip, 2005, 5, 536.

36 S. Kraszewski, M. Tarek and C. Ramseyer, ACS Nano, 2011, 5, 8571.

37 H. Ali-Boucetta, A. Nunes, R. Sainz, M.A. Herrero, B. Tian, M. Prato, A. Bianco and K. Kostarelos, Angew. Chem. Int. Ed., 2013, 52, 2274.

38 D. Pantarotto, J.-P. Briand, M. Prato and A. BiancoChem. Commun., 2004, 16.

39 L. Lacerda, G. Pastorin, W. Wu, M. Prato, A.Bianco and K. Kostarelos, Adv. Funct. Mater., 2006, 16, 1839.

40 R. Singh, D. Pantarotto, L. Lacerda, G. Pastorin, C. Klumpp, M. Prato, A.Bianco and K. Kostarelos, Proc. Natl. Acad. Sci. USA, 2006, 103, 3357.

41 J.C. Phillips, R. Braun, W. Wang, J. Gumbart, E. Tajkhorshid, E. Villa, C. Chipot, R.D. Skeel, L. Kalé and K. Schulten, J. Comput. Chem., 2005, 26, 1781.

42 S.E. Feller, Y. Zhang, R.W. Pastor and B.R. Brooks, J. Chem. Phys., 1995, 103, 4613.

43 T. Darden, D. York and L. Pedersen, J. Chem. Phys., 1993, 98, 10089.

44 N. Foloppe and A.D. MacKerell, J. Comput. Chem., 2000, 21, 86.

45 W.L. Jorgensen, J. Chandrasekhar, J.D. Madura, R.W. Impey and M.L. Klein, J. Chem. Phys., 1983, 79, 926.

46 L. W. Li, H. Davande, D. Bedrov and G.D. Smith, J. Phys. Chem. B, 2007, 111, 4067.

47 M.J. Frisch, G.W. Trucks, H.B. Schlegel, G.E. Scuseria, M.A. Robb et al. Gaussian, Inc.: Wallingford CT.

48 R.S. Mulliken, J. Chem. Phys., 1955, 23, 1833. 